\title{
Some ideal convergence of double $\Lambda$-interval number sequences defined by Orlicz function
}

\author{
Ayhan Esi ${ }^{1 *}$ and Bipan Hazarika ${ }^{2}$ \\ ${ }^{1}$ Adiyaman University, Science and Art Faculty, Department of Mathematics, 02040, Adiyaman, Turkey \\ ${ }^{2}$ Department of Mathematics, Rajiv Gandhi University, Rono Hills, Doimukh-791112. Arunachal Pradesh, India \\ *Corresponding author E-mail: aesi23@adiyaman.edu.tr
}

\begin{abstract}
An ideal $I$ is a family of subsets of positive integers $\mathbb{N}$ which is closed under taking finite unions and subsets of its elements. In this paper we introduce some ideal convergent double interval valued numbers sequence spaces defined by Orlicz function and study different properties of these spaces. We also establish some inclusion relations among them.
\end{abstract}

Keywords: Completeness, ideal-convergence, interval numbers, $\Lambda$-convergence, Orlicz function.

\section{Introduction}

Interval arithmetic was first suggested by Dwyer [2] in 1951. Development of interval arithmetic as a formal system and evidence of its value as a computational device was provided by Moore [16] in 1959 and Moore and Yang [17] in 1962. Further works on interval numbers can be found in Dwyer [3], Fischer [9], Markov [15]. Furthermore, Moore and Yang [18], have developed applications to differential equations.

Chiao in [1] introduced sequence of interval numbers and defined usual convergence of sequences of interval number. Şengönül and Eryilmaz in [24] introduced and studied bounded and convergent sequence spaces of interval numbers and showed that these spaces are complete metric space. Esi in [4], [5] introduced and studied strongly almost $\lambda$-convergence and statistically almost $\lambda$-convergence of interval numbers and lacunary sequence spaces of interval numbers, respectively. In [6], Esi and Hazarika introduced the difference classes of interval numbers. Recently Esi [7] has studied double sequences of interval numbers.

A set consisting of a closed interval of real numbers $x$ such that $a \leq x \leq b$ is called an interval number. A real interval can also be considered as a set. Thus we can investigate some properties of interval numbers, for instance arithmetic properties or analysis properties. We denote the set of all real valued closed intervals by IR. Any elements of $I \mathbb{R}$ is called closed interval and denoted by $\bar{x}$. That is $\bar{x}=\{x \in \mathbb{R}: a \leq x \leq b\}$. An interval number $\bar{x}$ is a closed subset of real numbers [1]. Let $x_{l}$ and $x_{r}$ be first and last points of $\bar{x}$ interval number, respectively. For $\bar{x}_{1}, \bar{x}_{2} \in \mathbb{I} \mathbb{R}$, we have $\bar{x}_{1}=\bar{x}_{2} \Leftrightarrow x_{1_{l}}=x_{2_{l}}, x_{1_{r}}=x_{2_{r}} . \bar{x}_{1}+\bar{x}_{2}=\left\{x \in \mathbb{R}: x_{1_{l}}+x_{2_{l}} \leq x \leq x_{1_{r}}+x_{2_{r}}\right\}$, and if $\alpha \geq 0$, then $\alpha \bar{x}=\left\{x \in \mathbb{R}: \alpha x_{1_{l}} \leq x \leq \alpha x_{1_{r}}\right\}$ and if $\alpha<0$, then $\alpha \bar{x}=\left\{x \in \mathbb{R}: \alpha x_{1_{r}} \leq x \leq \alpha x_{1_{l}}\right\}$,

$$
\bar{x}_{1} \cdot \bar{x}_{2}=\left\{x \in \mathbb{R}: \min \left\{x_{1_{l}} \cdot x_{2_{l}}, x_{1_{l}} \cdot x_{2_{r}}, x_{1_{r}} \cdot x_{2_{l}}, x_{1_{r}} . x_{2_{r}}\right\} \leq x \leq \max \left\{x_{1_{l}} \cdot x_{2_{l}}, x_{1_{l}} \cdot x_{2_{r}}, x_{1_{r}} . x_{2_{l}}, x_{1_{r}} \cdot x_{2_{r}}\right\}\right\} .
$$

In [16], Moore proved that the set of all interval numbers IR is a complete metric space defined by

$$
d\left(\bar{x}_{1}, \bar{x}_{2}\right)=\max \left\{\left|x_{1_{l}}-x_{2_{l}}\right|,\left|x_{1_{r}}-x_{2_{r}}\right|\right\} .
$$

In the special case $\bar{x}_{1}=[a, a]$ and $\bar{x}_{2}=[b, b]$, we obtain usual metric of $\mathbb{R}$.

Let us define transformation $f: \mathbb{N} \rightarrow \mathbb{R}$ by $k \rightarrow f(k)=\bar{x}, \bar{x}=\left(\bar{x}_{k}\right)$. Then $\bar{x}=\left(\bar{x}_{k}\right)$ is called sequence of interval numbers. The $\bar{x}_{k}$ is called $k^{t h}$ term of sequence $\bar{x}=\left(\bar{x}_{k}\right) \cdot w^{i}$ denotes the set of all interval numbers with real terms and the algebraic properties of $w^{i}$ can be found in [1].

Now we give the definition of convergence of interval numbers: 
Definition $1.1([1])$ A sequence $\bar{x}=\left(\bar{x}_{k}\right)$ of interval numbers is said to be convergent to the interval number $\bar{x}_{o}$ if for each $\varepsilon>0$ there exists a positive integer $k_{o}$ such that $d\left(\bar{x}_{k}, \bar{x}_{o}\right)<\varepsilon$ for all $k \geq k_{o}$ and we denote it by $\lim _{k} \bar{x}_{k}=\bar{x}_{o}$.

Thus, $\lim _{k} \bar{x}_{k}=\bar{x}_{o} \Leftrightarrow \lim _{k} x_{k_{l}}=x_{o_{l}}$ and $\lim _{k} x_{k_{r}}=x_{o_{r}}$.

The notion of $I$-convergence was initially introduced by Kostyrko, et. al [11] as a generalization of statistical convergence (see [8],[23] ) which is based on the structure of the ideal $I$ of subset of natural numbers $\mathbb{N}$. Kostyrko, et. al [12] gave some of basic properties of $I$-convergence and dealt with extremal $I$-limit points. Although an ideal is defined as a hereditary and additive family of subsets of a non-empty arbitrary set $X$, here in our study it suffices to take $I$ as a family of subsets of $\mathbb{N}$, positive integers, i.e. $I \subset 2^{\mathbb{N}}$, such that $A \cup B \in I$ for each $A, B \in I$, and each subset of an element of $I$ is an element of $I$.

A non-empty family of sets $F \subset 2^{\mathbb{N}}$ is a filter on $\mathbb{N}$ if and only if $\Phi \notin F, A \cap B \in F$ for each $A, B \in F$, and any subset of an element of $F$ is in $F$. An ideal $I$ is called non-trivial if $I \neq \Phi$ and $\mathbb{N} \notin I$. Clearly $I$ is a non-trivial ideal if and only if $F=F(I)=\{\mathbb{N}-A: A \in I\}$ is a filter in $\mathbb{N}$, called the filter associated with the ideal $I$. A non-trivial ideal $I$ is called admissible if and only if $\{\{n\}: n \in \mathbb{N}\} \subset I$. A non-trivial ideal $I$ is maximal if there cannot exist any non-trivial ideal $J \neq I$ containing $I$ as a subset. Further details on ideals can be found in Kostyrko, et.al (see [11]). Recall that a sequence $x=\left(x_{k}\right)$ of points in $\mathbb{R}$ is said to be $I$-convergent to a real number $\ell$ if $\left\{k \in \mathbb{N}:\left|x_{k}-\ell\right| \geq \varepsilon\right\} \in I$ for every $\varepsilon>0$ ([11]). In this case we write $I-\lim x_{k}=\ell$. Further details on ideal convergence can be found in [22], [27]. The notion of $I$-convergence double sequence was initially introduced by Tripathy and Tripathy (see $[26])$.

Let $\lambda=\left(\lambda_{m}\right)$ be a non-decreasing sequence of positive numbers tending to $\infty$ such that

$$
\lambda_{m+1} \leq \lambda_{m}+1, \lambda_{1}=1 \text {. }
$$

The generalized de la Vallée Poussin mean is defined by

$$
t_{m}(x)=\frac{1}{\lambda_{m}} \sum_{k \in J_{m}} x_{k}
$$

where $J_{m}=\left[m-\lambda_{m}+1, m\right]$.

A sequence $x=\left(x_{k}\right)$ is said to be $(V, \lambda)$-summable to a number $\ell$ (see [14] ) if

$$
t_{m}(x) \rightarrow \ell, \text { as } m \rightarrow \infty \text {. }
$$

If $\lambda_{m}=m$, then $(V, \lambda)$-summability reduces to $(C, 1)$-summability. We write

$$
[C, 1]=\left\{x=\left(x_{k}\right)\left|\exists \ell \in \mathbb{R}, \lim _{m \rightarrow \infty} \frac{1}{m} \sum_{k=1}^{m}\right| x_{k}-\ell \mid=0\right\}
$$

and

$$
[V, \lambda]=\left\{x=\left(x_{k}\right)\left|\exists \ell \in \mathbb{R}, \lim _{m \rightarrow \infty} \frac{1}{\lambda_{m}} \sum_{k \in J_{m}}\right| x_{k}-\ell \mid=0\right\}
$$

for the sets of sequences $x=\left(x_{k}\right)$ which are strongly Cesàro summable (see [10]) and strongly $(V, \lambda)$-summable to $\ell$, i.e. $x_{k} \rightarrow \ell[C, 1]$ and $x_{k} \rightarrow \ell[V, \lambda]$, respectively.

Let $\lambda=\left(\lambda_{m}\right)$ and $\mu=\left(\mu_{n}\right)$ be two non-decreasing sequences of positive real numbers, each tending to $\infty$ and such that $\lambda_{m+1} \leq \Delta \lambda_{m}+1, \lambda_{1}=1 ; \mu_{n+1} \leq \mu_{n}+1, \mu_{1}=1$. Let $J_{m}=\left[m-\lambda_{m}+1, m\right], J_{n}=\left[n-\mu_{n}+1, n\right], J_{m n}=J_{m} \times J_{n}$ and $\Lambda=\lambda_{m n}=\lambda_{m} \mu_{n}$.

We define the generalized double de la Vallée Poussin mean by

$$
t_{m n}(x)=\frac{1}{\lambda_{m n}} \sum_{(k, l) \in J_{m n}} x_{k, l} .
$$

Recall in [19],[13] that an Orlicz function $M$ is continuous, convex, nondecreasing function define for $x>0$ such that $M(0)=0$ and $M(x)>0$. If convexity of Orlicz function is replaced by $M(x+y) \leq M(x)+M(y)$ then this function is called the modulus function and characterized by Ruckle [21]. An Orlicz function $M$ is said to satisfy 
$\Delta_{2}-$ condition for all values $u$, if there exists $K>0$ such that $M(2 u) \leq K M(u), u \geq 0$. Subsequently, the notion of Orlicz function was used to defined sequence spaces by Tripathy et al [25], Tripathy and Hazarika[28] and many others.

An interval valued double sequence $\bar{x}=\left(\bar{x}_{k, l}\right)$ is said to be convergent in the Pringsheim's sense or $P$-convergent to an interval number $\bar{x}_{o}$, if for every $\varepsilon>0$, there exists $N \in \mathbb{N}$ such that

$$
d\left(\bar{x}_{k, l}, \bar{x}_{o}\right)<\varepsilon \text { for } k, l>N
$$

(see[20]) and we denote it by $P-\lim \bar{x}_{k, l}=\bar{x}_{o}$, where $d\left(\bar{x}_{k, l}, \bar{y}_{k, l}\right)$ is the Hausdorff distance between $\bar{x}=\left(\bar{x}_{k, l}\right)$ and $\bar{y}=\left(\bar{y}_{k, l}\right)$. The interval number $\bar{x}_{o}$ is called the Pringsheim limit of $\bar{x}=\left(\bar{x}_{k, l}\right)$. More exactly, we say that a double sequence of interval numbers $\bar{x}=\left(\bar{x}_{k, l}\right)$ converges to a finite interval number $\bar{x}_{o}$ if $\bar{x}_{k, l}$ tends to $\bar{x}_{o}$ as both $k$ and $l$ tend to infinity independently of each another. We denote by $\bar{c}^{2}$ the set of all double convergent interval numbers of double interval numbers.

The interval number double sequence $\bar{x}=\left(\bar{x}_{k, l}\right)$ is bounded if and only if there exists a positive number $B$ such that $d\left(\bar{x}_{k, l}, \overline{0}\right)<B$ for all $k$ and $l$. We shall denote all bounded interval number double sequences by $\bar{l}_{\infty}^{2}$. Let $\bar{w}^{2}$ denote the set of all double sequences of interval numbers.

Let $p=\left(p_{i, j}\right)$ be a double sequence of positive real numbers. If $0<p_{i, j} \leq \sup _{i, j} p_{i, j}=H<\infty$ and $D=$ $\max \left(1,2^{H-1}\right)$, then for $a_{i, j}, b_{i, j} \in \mathbb{R}$ for all $i, j \in \mathbb{N}$, we have

$$
\left|a_{i, j}+b_{i, j}\right|^{p_{i, j}} \leq D\left(\left|a_{i, j}\right|^{p_{i, j}}+\left|b_{i, j}\right|^{p_{i, j}}\right) .
$$

\section{Main results}

In this paper, we define new double sequence spaces for interval sequences as follows.

Let $\mathcal{I}$ be an admissible ideal of $\mathbb{N} \times \mathbb{N}$. Let $M$ be an Orlicz function and $p=\left(p_{i, j}\right)$ be a double sequence of strictly positive real numbers. We introduce the following sequence spaces:

$$
\begin{array}{r}
{ }_{2} \bar{w}^{\mathcal{I}}(\Lambda, M, p)=\left\{\begin{array}{r}
\bar{x}=\left(\bar{x}_{i, j}\right):\left\{(m, n) \in \mathbb{N} \times \mathbb{N}: \frac{1}{\lambda_{m n}} \sum_{(i, j) \in J_{m n}}\left[M\left(\frac{d\left(\bar{x}_{i, j}, \bar{x}_{o}\right)}{\rho}\right)\right]^{p_{i, j}} \geq \varepsilon\right\} \in \mathcal{I}, \\
\text { for some } \rho>0, \text { and } \bar{x}_{o} \in \mathbf{I R}
\end{array}\right\}, \\
{ }_{2} \bar{w}_{o}^{\mathcal{I}}(\Lambda, M, p)=\left\{\begin{array}{c}
\bar{x}=\left(\bar{x}_{i, j}\right):\left\{(m, n) \in \mathbb{N} \times \mathbb{N}: \frac{1}{\lambda_{m n}} \sum_{(i, j) \in J_{m n}}\left[M\left(\frac{d\left(\bar{x}_{i, j}, \overline{0}\right)}{\rho}\right)\right]^{p_{i, j}} \geq \varepsilon\right\} \in \mathcal{I}, \\
\text { for some } \rho>0
\end{array}\right\} \\
{ }_{2} \bar{w}_{\infty}^{\mathcal{I}}(\Lambda, M, p)=\left\{\begin{array}{c}
\bar{x}=\left(\bar{x}_{i, j}\right): \exists K>0 \text { s.t. }\left\{(m, n) \in \mathbb{N} \times \mathbb{N}: \frac{1}{\lambda_{m n}} \sum_{(i, j) \in J_{m n}}\left[M\left(\frac{d\left(\bar{x}_{i, j}, \overline{0}\right)}{\rho}\right)\right]^{p_{i, j}} \geq K\right\} \in \mathcal{I}, \\
\text { for some } \rho>0
\end{array}\right\} .
\end{array}
$$

and

$$
{ }_{2} \bar{w}_{\infty}(\Lambda, M, p)=\left\{\bar{x}=\left(\bar{x}_{i, j}\right): \sup _{m, n} \frac{1}{\lambda_{m n}} \sum_{(i, j) \in J_{m n}}\left[M\left(\frac{d\left(\bar{x}_{i, j}, \overline{0}\right)}{\rho}\right)\right]^{p_{i, j}}<\infty, \text { for some } \rho>0\right\} .
$$

The proof of the following result is a routine work, so we omit it.

Proposition 2.1 (a) ${ }_{2} \bar{w}^{\mathcal{I}}(\Lambda, M, p) \subset{ }_{2} \bar{w}_{\infty}(\Lambda, M, p)$,

(b) ${ }_{2} \bar{w}_{o}^{\mathcal{I}}(\Lambda, M, p) \subset{ }_{2} \bar{w}_{\infty}(\Lambda, M, p)$.

Theorem 2.2 (a) If $0<\inf _{i, j} p_{i, j} \leq p_{i, j}<1$, then ${ }_{2} \bar{w}^{\mathcal{I}}(\Lambda, M, p) \subset{ }_{2} \bar{w}^{\mathcal{I}}(\Lambda, M)$,

(b) If $1<p_{i, j}<\sup _{i, j} p_{i, j}<\infty$, then ${ }_{2} \bar{w}^{\mathcal{I}}(\Lambda, M) \subset{ }_{2} \bar{w}^{\mathcal{I}}(\Lambda, M, p)$,

(c) If $0<p_{i, j} \leq q_{i, j}<\infty$ and $\left(\frac{q_{i, j}}{p_{i, j}}\right)$ is bounded, then ${ }_{2} \bar{w}^{\mathcal{I}}(\Lambda, M, p) \subset{ }_{2} \bar{w}^{\mathcal{I}}(\Lambda, M, q)$.

Proof. The first part of the result follows from the relation

$$
\begin{aligned}
\left\{(m, n) \in \mathbb{N} \times \mathbb{N}: \frac{1}{\lambda_{m n}} \sum_{(i, j) \in J_{m n}} M\left(\frac{d\left(\bar{x}_{i, j}, \bar{x}_{o}\right)}{\rho}\right) \geq \varepsilon\right\} & \\
& \subseteq\left\{(m, n) \in \mathbb{N} \times \mathbb{N}: \frac{1}{\lambda_{m n}} \sum_{(i, j) \in J_{m n}}\left[M\left(\frac{d\left(\bar{x}_{i, j}, \bar{x}_{o}\right)}{\rho}\right)\right]^{p_{i, j}} \geq \varepsilon\right\}
\end{aligned}
$$


and the second part of the result follows from the relation

$$
\begin{aligned}
\left\{(m, n) \in \mathbb{N} \times \mathbb{N}: \frac{1}{\lambda_{m n}} \sum_{(i, j) \in J_{m n}}\left[M\left(\frac{d\left(\bar{x}_{i, j}, \bar{x}_{o}\right)}{\rho}\right)\right]^{p_{i, j}} \geq \varepsilon\right\} & \\
& \subseteq\left\{(m, n) \in \mathbb{N} \times \mathbb{N}: \frac{1}{\lambda_{m n}} \sum_{(i, j) \in J_{m n}} M\left(\frac{d\left(\bar{x}_{i, j}, \bar{x}_{o}\right)}{\rho}\right) \geq \varepsilon\right\}
\end{aligned}
$$

This completes the proof.

The proof of the part three is easy, so omitted.

Theorem 2.3 (a) If $0<\inf _{i, j} p_{i, j} \leq p_{i, j}<1$, then ${ }_{2} \bar{w}_{0}^{\mathcal{I}}(\Lambda, M, p) \subset{ }_{2} \bar{w}_{0}^{\mathcal{I}}(\Lambda, M)$,

(b) If $1<p_{i, j}<\sup _{i, j} p_{i, j}<\infty$, then ${ }_{2} \bar{w}_{0}^{\mathcal{I}}(\Lambda, M) \subset{ }_{2} \bar{w}_{0}^{\mathcal{I}}(\Lambda, M, p)$,

(c) If $0<p_{i, j} \leq q_{i, j}<\infty$ and $\left(\frac{q_{i, j}}{p_{i, j}}\right)$ is bounded, then ${ }_{2} \bar{w}_{0}^{\mathcal{I}}(\Lambda, M, p) \subset{ }_{2} \bar{w}_{0}^{\mathcal{I}}(\Lambda, M, q)$.

Proof of the result follows from the Theorem 2.2.

Theorem 2.4 Let $M_{1}$ and $M_{2}$ be two Orlicz functions. Then

$$
{ }_{2} \bar{w}^{\mathcal{I}}\left(\Lambda, M_{1}, p\right) \cap{ }_{2} \bar{w}^{\mathcal{I}}\left(\Lambda, M_{2}, p\right) \subset{ }_{2} \bar{w}^{\mathcal{I}}\left(\Lambda, M_{1}+M_{2}, p\right) .
$$

Proof. Let $\left(\bar{x}_{i, j}\right) \in{ }_{2} \bar{w}^{\mathcal{I}}\left(\Lambda, M_{1}, p\right) \cap{ }_{2} \bar{w}^{\mathcal{I}}\left(\Lambda, M_{2}, p\right)$. Then for every $\varepsilon>0$ we have

$$
\left\{(m, n) \in \mathbb{N} \times \mathbb{N}: \frac{1}{\lambda_{m n}} \sum_{(i, j) \in J_{m n}}\left[M_{1}\left(\frac{d\left(\bar{x}_{i, j}, \bar{x}_{o}\right)}{\rho_{1}}\right)\right]^{p_{i, j}} \geq \varepsilon\right\} \in \mathcal{I}, \text { for some } \rho_{1}>0
$$

and

$$
\left\{(m, n) \in \mathbb{N} \times \mathbb{N}: \frac{1}{\lambda_{m n}} \sum_{(i, j) \in J_{m n}}\left[M_{2}\left(\frac{d\left(\bar{x}_{i, j}, \bar{x}_{o}\right)}{\rho_{2}}\right)\right]^{p_{i, j}} \geq \varepsilon\right\} \in \mathcal{I}, \text { for some } \rho_{2}>0 .
$$

Let $\rho=\max \left\{\rho_{1}, \rho_{2}\right\}$. The result follows from the following inequality

$$
\begin{aligned}
& \sum_{(i, j) \in J_{m n}}\left[\left(M_{1}+M_{2}\right)\left(\frac{d\left(\bar{x}_{i, j}, \bar{x}_{o}\right)}{\rho}\right)\right]^{p_{i, j}} \\
& \leq D\left(\sum_{(i, j) \in J_{m n}}\left[M_{1}\left(\frac{d\left(\bar{x}_{i, j}, \bar{x}_{o}\right)}{\rho_{1}}\right)\right]^{p_{i, j}}+\sum_{(i, j) \in J_{m n}}\left[M_{2}\left(\frac{d\left(\bar{x}_{i, j}, \bar{x}_{o}\right)}{\rho_{2}}\right)\right]^{p_{i, j}}\right)
\end{aligned}
$$

This completes the proof.

Theorem 2.5 Let $M_{1}$ and $M_{2}$ be two Orlicz functions. Then

$$
{ }_{2} \bar{w}^{\mathcal{I}}\left(\Lambda, M_{1}, p\right) \subset_{2} \bar{w}^{\mathcal{I}}\left(\Lambda, M_{2} \circ M_{1}, p\right) .
$$

Proof. Let $\inf p_{i, j}=H_{0}$. For given $\varepsilon>0$, we first choose $\varepsilon_{0}>0$ such that $\max \left\{\varepsilon_{0}^{H}, \varepsilon_{0}^{H_{0}}\right\}<\varepsilon$. Now using the continuity of $M_{2}$ choose $0<\delta<1$ such that $0<t<\delta$ implies $M_{2}(t)<\varepsilon_{0}$. Let $\left(\bar{x}_{i, j}\right) \in{ }_{2} \bar{w}^{\mathcal{I}}\left(\Lambda, M_{1}, p\right)$. Now from the definition of ${ }_{2} \bar{w}^{\mathcal{I}}\left(\Lambda, M_{1}, p\right)$, for some $\rho>0$

$$
\bar{A}(\delta)=\left\{(m, n) \in \mathbb{N} \times \mathbb{N}: \frac{1}{\lambda_{m n}} \sum_{(i, j) \in J_{m n}}\left[M_{1}\left(\frac{d\left(\bar{x}_{i, j}, \bar{x}_{o}\right)}{\rho}\right)\right]^{p_{i, j}} \geq \delta^{H}\right\} \in \mathcal{I} .
$$


Thus if $(m, n) \notin \bar{A}(\delta)$ then we have

$$
\begin{aligned}
& \frac{1}{\lambda_{m n}} \sum_{(i, j) \in J_{m n}}\left[M_{1}\left(\frac{d\left(\bar{x}_{i, j}, \bar{x}_{o}\right)}{\rho}\right)\right]^{p_{i, j}}<\delta^{H} \\
\Rightarrow & \sum_{(i, j) \in J_{m n}}\left[M_{1}\left(\frac{d\left(\bar{x}_{i, j}, \bar{x}_{o}\right)}{\rho}\right)\right]^{p_{i, j}}<\lambda_{m n} \delta^{H} \\
\Rightarrow & {\left[M_{1}\left(\frac{d\left(\bar{x}_{i, j}, \bar{x}_{o}\right)}{\rho}\right)\right]^{p_{i, j}}<\delta^{H}, \text { for all } i, j=1,2,3 \ldots } \\
\Rightarrow & M_{1}\left(\frac{d\left(\bar{x}_{i, j}, \bar{x}_{o}\right)}{\rho}\right)<\delta, \text { for all } i, j=1,2,3 \ldots
\end{aligned}
$$

Hence from above inequality and using continuity of $M_{2}$, we must have

$$
M_{2}\left(M_{1}\left(\frac{d\left(\bar{x}_{i, j}, \bar{x}_{o}\right)}{\rho}\right)\right)<\varepsilon_{0}, \text { for all } i, j=1,2,3 \ldots
$$

which consequently implies that

$$
\begin{aligned}
& \sum_{(i, j) \in J_{m n}}\left[M_{2}\left(M_{1}\left(\frac{d\left(\bar{x}_{i, j}, \bar{x}_{o}\right)}{\rho}\right)\right)\right]^{p_{i, j}}<\lambda_{m n} \max \left\{\varepsilon_{0}^{H}, \varepsilon_{0}^{H_{0}}\right\}<\lambda_{m n} \varepsilon \\
\Rightarrow & \frac{1}{\lambda_{m n}} \sum_{(i, j) \in J_{m n}}\left[M_{2}\left(M_{1}\left(\frac{d\left(\bar{x}_{i, j}, \bar{x}_{o}\right)}{\rho}\right)\right)\right]^{p_{i, j}}<\varepsilon .
\end{aligned}
$$

This shows that

$$
\left\{(m, n) \in \mathbb{N} \times \mathbb{N}: \frac{1}{\lambda_{m n}} \sum_{(i, j) \in J_{m n}}\left[M_{2}\left(M_{1}\left(\frac{d\left(\bar{x}_{i, j}, \bar{x}_{o}\right)}{\rho}\right)\right)\right]^{p_{i, j}} \geq \varepsilon\right\} \subset \bar{A}(\delta)
$$

and so belongs to $\mathcal{I}$. This completes the proof.

Theorem 2.6 Let $M_{1}$ and $M_{2}$ be two Orlicz functions. Then

(a) ${ }_{2} \bar{w}_{0}^{\mathcal{I}}\left(\Lambda, M_{1}, p\right) \cap{ }_{2} \bar{w}_{0}^{\mathcal{I}}\left(\Lambda, M_{2}, p\right) \subset{ }_{2} \bar{w}_{0}^{\mathcal{I}}\left(\Lambda, M_{1}+M_{2}, p\right)$;

(b) ${ }_{2} \bar{w}_{0}^{\mathcal{I}}\left(\Lambda, M_{1}, p\right) \subset{ }_{2} \bar{w}_{0}^{\mathcal{I}}\left(\Lambda, M_{2} \circ M_{1}, p\right)$.

The proof of the theorem follows from the Theorems 2.4 and 2.5.

Theorem 2.7 Let $M_{1}$ and $M_{2}$ be two Orlicz functions satisfying $\Delta_{2}$-condition. If $\beta=\lim _{t \rightarrow \infty} \frac{M_{2}(t)}{t} \geq 1$, then (a) ${ }_{2} \bar{w}_{0}^{\mathcal{I}}\left(\Lambda, M_{1}, p\right)={ }_{2} \bar{w}_{0}^{\mathcal{I}}\left(\Lambda, M_{2} \circ M_{1}, p\right)$,

(b) $)_{2} \bar{w}^{\mathcal{I}}\left(\Lambda, M_{1}, p\right)={ }_{2} \bar{w}^{\mathcal{I}}\left(\Lambda, M_{2} \circ M_{1}, p\right)$.

Proof. It is easy, so omitted.

Two non-negative functions $f, g$ are called equivalent, whenever $C_{1} f \leq g \leq C_{2} f$, for some $C_{j}>0, j=1,2$ and in this case we write $f \approx g$.

The following Theorem is a direct consequence of definition of equivalent mappings.

Theorem 2.8 Let $M_{1}$ and $M_{2}$ be two Orlicz functions such that $M_{1} \approx M_{2}$. Then $Z\left(\Lambda, M_{1}, p\right)=Z\left(\Lambda, M_{2}, p\right)$, for $Z={ }_{2} \bar{w}_{0}^{\mathcal{I}},{ }_{2} \bar{w}^{\mathcal{I}},{ }_{2} \bar{w}_{\infty}^{\mathcal{I}}$ and ${ }_{2} \bar{w}_{\infty}$.

A sequence space $E$ is said to be solid (or normal) if $\left(x_{k}\right) \in E$ and for all sequence $\left(\alpha_{k}\right)$ of scalars with $\left|\alpha_{k}\right| \leq 1$, for all $k \in \mathbb{N}$ implies $\left(\alpha_{k} x_{k}\right) \in E$.

Let $K=\left\{k_{1}<k_{2}<\ldots\right\} \subseteq \mathbb{N}$ and $E$ be a sequence space. A $K$-step space of $E$ is a sequence $\operatorname{space} \lambda_{K}^{E}=$ $\left\{\left(x_{k_{n}}\right) \in w:\left(k_{n}\right) \in E\right\}$.

A canonical preimage of a sequence $\left(x_{k_{n}}\right) \in \lambda_{K}^{E}$ is a sequence $\left(y_{n}\right) \in w$ defined as

$$
y_{n}=\left\{\begin{array}{cc}
x_{n}, & \text { if } n \in \mathbb{N} ; \\
0, & \text { otherwise }
\end{array}\right.
$$

A canonical preimage of a step space $\lambda_{K}^{E}$ is a set of canonical preimages of all elements in $\lambda_{K}^{E}$, i.e. $y$ is in canonical preimage of $\lambda_{K}^{E}$ if and only if $y$ is canonical preimage of some $x \in \lambda_{K}^{E}$.

A sequence space $E$ is said to be monotone if it contains the canonical preimages of its step spaces. 
Lemma 2.9 Every normal space is monotone.

Theorem 2.10 The double sequence space ${ }_{2} \bar{w}^{\mathcal{I}}(\Lambda, M, p),{ }_{2} \bar{w}_{o}^{\mathcal{I}}(\Lambda, M, p),{ }_{2} \bar{w}_{\infty}^{\mathcal{I}}(\Lambda, M, p)$ and ${ }_{2} \bar{w}_{\infty}(\Lambda, M, p)$ are solid as well as monotone.

Proof. We give the proof for only ${ }_{2} \bar{w}_{o}^{\mathcal{I}}(\Lambda, M, p)$. The others can be proved similarly. Let $\bar{x}=\left(\bar{x}_{i, j}\right) \in{ }_{2} \bar{w}_{o}^{\mathcal{I}}(\Lambda, M, p)$ and $\left(\alpha_{i, j}\right)$ be a scalar sequence such that $\left|\alpha_{i, j}\right| \leq 1$ for all $i, j \in \mathbb{N}$. Then for every $\varepsilon>0$ we have

$$
\begin{aligned}
\left\{(m, n) \in \mathbb{N} \times \mathbb{N}: \frac{1}{\lambda_{m n}} \sum_{(i, j) \in J_{m n}}\left[M\left(\frac{d\left(\alpha_{i, j} \bar{x}_{i, j}, \overline{0}\right)}{\rho}\right)\right]^{p_{i, j}} \geq \varepsilon\right\} \\
\subseteq\left\{(m, n) \in \mathbb{N} \times \mathbb{N}: \frac{E}{\lambda_{m n}} \sum_{(i, j) \in J_{m n}}\left[M\left(\frac{d\left(\bar{x}_{i, j}, \overline{0}\right)}{\rho}\right)\right]^{p_{i, j}} \geq \varepsilon\right\} \in \mathcal{I},
\end{aligned}
$$

where $E=\max \left\{1,\left|\alpha_{k, l}\right|^{H}\right\}$. Hence $(\alpha \bar{x}) \in{ }_{2} \bar{w}_{o}^{\mathcal{I}}(\Lambda, M, p)$. By Lemma 1 , the space ${ }_{2} \bar{w}_{o}^{\mathcal{I}}(\Lambda, M, p)$ is monotone. This completes the proof.

\section{$3 \mathcal{I}_{\Lambda}$-convergence of interval numbers}

Definition 3.1 A double sequence $\bar{x}=\left(\bar{x}_{i, j}\right)$ of interval numbers is said to be $\Lambda$-convergent to $\bar{x}_{0} \in \mathbf{I R}$ if for every $\varepsilon>0$, there exists a positive integer $N$ such that

$$
d\left(t_{m n}(\bar{x}), \bar{x}_{0}\right)<\varepsilon \text { for all } m, n>N \text {. }
$$

Definition $3.2 A$ double sequence $\bar{x}=\left(\bar{x}_{i, j}\right)$ of interval numbers is said to be $\mathcal{I}_{\Lambda}$-convergent to $\bar{x}_{0} \in \mathbf{I R}$ if for every $\varepsilon>0$, the set

$$
\bar{K}_{\varepsilon}(\Lambda)=\left\{(m, n) \in \mathbb{N} \times \mathbb{N}: d\left(t_{m n}(\bar{x}), \bar{x}_{0}\right) \geq \varepsilon\right\} \in \mathcal{I} .
$$

or equivalently

$$
\left\{(m, n) \in \mathbb{N} \times \mathbb{N}: d\left(t_{m n}(\bar{x}), \bar{x}_{0}\right)<\varepsilon\right\} \in F(\mathcal{I}) .
$$

In this case we write $\mathcal{I}_{\Lambda}-\lim \bar{x}=\bar{x}_{0}$.

Theorem 3.3 Let $\bar{x}=\left(\bar{x}_{i, j}\right)$ be a double sequence in IR. If $\Lambda-\lim \bar{x}=\bar{x}_{0}$, then $\mathcal{I}_{\Lambda}-\lim \bar{x}=\bar{x}_{0}$.

Proof. Let $\Lambda-\lim \bar{x}=\bar{x}_{0}$, then for every $\varepsilon>0$, there exists $N \in \mathbb{N}$ such that

$$
d\left(t_{m n}(\bar{x}), \bar{x}_{0}\right)<\varepsilon, \text { for all } m, n>N .
$$

Therefore the set

$$
B=\left\{(m, n) \in \mathbb{N}: d\left(t_{m n}(\bar{x}), \bar{x}_{0}\right) \geq \varepsilon\right\} \subseteq\{(1,1),(2,2), \ldots,(N-1, N-1)\} .
$$

But, $\mathcal{I}$ being admissible, we have $B \in \mathcal{I}$. Hence $\mathcal{I}_{\Lambda}-\lim \bar{x}=\bar{x}_{0}$.

Theorem 3.4 Sequential method $\mathcal{I}_{\Lambda}$ is regular.

Proof. The proof follows from the fact that $\mathcal{I}$ is admissible and Theorem 3.3.

\section{References}

[1] Kuo-Ping Chiao, Fundamental properties of interval vector max-norm, Tamsui Oxford Journal of Mathematics, 18(2)(2002), 219-233.

[2] P. S. Dwyer, Linear Computation, New York, Wiley, 1951. 
[3] P. S. Dwyer, Errors of matrix computation, simultaneous equations and eigenvalues, National Bureu of Standarts, Applied Mathematics Series, 29(1953), 49-58.

[4] A. Esi, Strongly almost $\lambda$-convergence and statistically almost $\lambda$-convergence of interval numbers, Scientia Magna, $7(2)(2011), 117-122$.

[5] A. Esi, Lacunary convergence of interval numbers, Thai Journal of Mathematics,10(2)(2012), 445-451.

[6] A. Esi, B. Hazarika, On Interval Valued Generalized Difference Classes Defined By Orlicz Function, (Submitted for publication).

[7] A. Esi, Some double sequence spaces of interval numbers defined by Orlicz functions, Acta et Commentationes Universitatis Tartuensis de Mathematica, 17(1)(2013), 57-64.

[8] H. Fast, Sur la convergence statistique, Collog.Math.2 (1951), 241-244.

[9] P. S. Fischer, Automatic propagated and round-off error analysis, paper presented at the 13th National Meeting of the Association of Computing Machinery, June 1958.

[10] A. R. Freedman, J. J. Sember, M. Raphael, Some Cesàro-type summability spaces, Proc. London Math. Soc., 37(3) (1978) 508-520.

[11] P. Kostyrko, T. Salát, and W. Wilczyński, I-convergence, Real Anal. Exchange 26, 2, (2000-2001),669-686, MR 2002e:54002.

[12] P. Kostyrko, M. Macaj, T. S̆alat, M. Sleziak, I-convergence and Extremal I-limit points, Math. Slovaca 2005; 55; $443-64$.

[13] M. A. Krasnoselski and Y. B. Rutickii, Convex function and Orlicz spaces, Groningen, Nederland, 1961.

[14] L. Leindler, Über die de la Vallée Pousinsche Summierbarkeit allgenmeiner Othogonalreihen, Acta Math. Acad. Sci. Hungar, 16(1965), 375-387.

[15] S. Markov, Quasilinear spaces and their relation to vector spaces, Electronic Journal on Mathematics of Computation, $2(1)(2005)$.

[16] R. E. Moore, Automatic Error Analysis in Digital Computation, LSMD-48421, Lockheed Missiles and Space Company, 1959.

[17] R. E. Moore and C. T. Yang, Interval Analysis I, LMSD-285875, Lockheed Missiles and Space Company, 1962.

[18] R. E. Moore and C. T. Yang, Theory of an interval algebra and its application to numeric analysis, RAAG Memories II, Gaukutsu Bunken Fukeyu-kai, Tokyo, 1958.

[19] H. Nakano, Concave modulars, J.Math.Soc.Japan, 5(1953), 29-49.

[20] A. Pringsheim, Zur theorie der zweifach unendlichen zahlenfolgen, Math. Ann. Soc.,53(1900), 289-321.

[21] W. H. Ruckle, FK-spaces in which the sequence of coordinate vectors is bounded, Canad.J.Math.,25(1973), 973-978.

[22] T. Salát, B. C. Tripathy, M. Ziman, On some properties of I-convergence, Tatra Mt. Math. Publ. 2004; $28 ; 279-86$.

[23] I. J. Schoenberg, The integrability of certain functions and related summability methods, Amer.Math.Monthly, 66(1959), 361-375.

[24] M. Şengönül and A. Eryılmaz, On the sequence spaces of interval numbers, Thai Journal of Mathematics, 8(3)(2010), 503-510.

[25] B. C. Tripathy, Y. Altin and M. Et, Generalized difference sequence spaces on seminormed spaces defined by Orlicz functions, Mathematica Slovaca, 58(3)(2008), 315-324.

[26] B. K. Tripathy, B. C. Tripathy, On I-convergent double sequences, Soochow J.Math., 31(4) (2005) 549-560.

[27] B. C. Tripathy, B. Hazarika, I-monotonic and I-convergent sequences, Kyungpook Math. J. 51(2011), 233-239, DOI 10.5666-KMJ.2011.51.2.233

[28] B. C. Tripathy, B. Hazarika, Some I-convergent sequence spaces defined by Orlicz functions, Acta Math. Appl. Sinica, 27(1) (2011) 149-154. 